\title{
A spatially explicit hierarchical model to characterize population viability
}

\author{
Steven P. Campbell, ${ }^{1,4,5}$ Erin R. Zylstra iD,${ }^{1}$ Catherine R. Darst, ${ }^{2}$ Roy C. Averill-Murray, ${ }^{3}$ and Robert J. Steidl iD 1 \\ ${ }^{1}$ School of Natural Resources and the Environment, University of Arizona, Tucson, Arizona 85721 USA \\ ${ }^{2}$ Desert Tortoise Recovery Office, U. S. Fish and Wildlife Service, Ventura, California 93003 USA \\ ${ }^{3}$ Desert Tortoise Recovery Office, U. S. Fish and Wildlife Service, Reno, Nevada 89502 USA
}

\begin{abstract}
Many of the processes that govern the viability of animal populations vary spatially, yet population viability analyses (PVAs) that account explicitly for spatial variation are rare. We develop a PVA model that incorporates autocorrelation into the analysis of local demographic information to produce spatially explicit estimates of demography and viability at relatively fine spatial scales across a large spatial extent. We use a hierarchical, spatial, autoregressive model for capture-recapture data from multiple locations to obtain spatially explicit estimates of adult survival $\left(\phi_{\mathrm{ad}}\right)$, juvenile survival $\left(\phi_{\mathrm{juv}}\right)$, and juvenile-to-adult transition rates $(\psi)$, and a spatial autoregressive model for recruitment data from multiple locations to obtain spatially explicit estimates of recruitment $(R)$. We combine local estimates of demographic rates in stage-structured population models to estimate the rate of population change $(\lambda)$, then use estimates of $\lambda$ (and its uncertainty) to forecast changes in local abundance and produce spatially explicit estimates of viability (probability of extirpation, $P_{\mathrm{ex}}$ ). We apply the model to demographic data for the Sonoran desert tortoise (Gopherus morafkai) collected across its geographic range in Arizona. There was modest spatial variation in $\hat{\lambda}(0.94-1.03)$, which reflected spatial variation in $\hat{\phi}_{\mathrm{ad}}(0.85-0.95), \hat{\phi}_{\mathrm{juv}}(0.70-0.89)$, and $\hat{\psi}(0.07-0.13)$. Recruitment data were too sparse for spatially explicit estimates; therefore, we used a range-wide estimate $(\hat{R}=0.321$-yr-old females per female per year). Spatial patterns in demographic rates were complex, but $\hat{\phi}_{\mathrm{ad}}, \hat{\phi}_{\mathrm{juv}}$, and $\hat{\lambda}$ tended to be lower and $\hat{\psi}$ higher in the northwestern portion of the range. Spatial patterns in $P_{\text {ex }}$ varied with local abundance. For local abundances $>500, P_{\mathrm{ex}}$ was near zero $(<0.05)$ across most of the range after $100 \mathrm{yr}$; as abundances decreased, however, $P_{\mathrm{ex}}$ approached one in the northwestern portion of the range and remained low elsewhere. When local abundances were $<50$, western and southern populations were vulnerable $\left(P_{\mathrm{ex}}>0.25\right)$. This approach to PVA offers the potential to reveal spatial patterns in demography and viability that can inform conservation and management at multiple spatial scales, provide insight into scale-related investigations in population ecology, and improve basic ecological knowledge of landscape-level phenomena.
\end{abstract}

Key words: capture-recapture; CAR model; demography; Gopherus morafkai; multi-state model; population viability analysis; recruitment; Sonoran desert tortoise; spatial autoregressive model; spatial variation; survival.

\section{INTRODUCTION}

Population viability analysis (PVA) is a broad collection of strategies to forecast the status of a population or a collection of populations (Beissinger and McCullough 2002). Typically, PVAs integrate information on demographic processes and their variability into stochastic simulation models that are used to project population trends and estimate the risk of extinction or some other measure of viability (Morris and Doak 2002, Ralls et al. 2002, Reed et al. 2002). PVAs are used commonly to understand the population biology of rare and threatened species and to provide information to develop strategies to minimize extinction risk (Beissinger and Westphal 1998, Menges 2000, Ralls et al. 2002). Within this context, PVAs have been used to explore the contribution of different demographic rates to population viability, to assess the impacts of human activities, to contrast management alternatives, to establish recovery criteria, and to guide research (Schemske et al. 1994, Carroll et al. 1996, Akçakaya and Sjögren-Gulve 2000).

Manuscript received 2 November 2017; revised 21 June 2018; accepted 30 July 2018. Corresponding Editor: Trenton W. J. Garner.

${ }^{4}$ Present address: Albany Pine Bush Preserve Commission, Albany, New York 12205 USA

${ }^{5}$ E-mail: scampbell@albanypinebush.org
PVA is a relatively well-established tool for assessing extinction risks at local scales (e.g., a single population) where the demographic processes that govern viability—survival, individual growth (i.e., transitions between life stages), and reproduction - are unlikely to vary markedly across the area of interest (Beissinger and Westphal 1998, Morris and Doak 2002). At larger spatial scales (e.g., multiple populations or a species range), however, its utility and reliability depend on the ability to account for spatial variation in demographic processes that is common across larger scales (Ozgul et al. 2006, Hernández-Matías et al. 2013, Zeigler et al. 2013, Zylstra et al. 2013). Generally, PVAs have confronted spatial variation at large scales in three different ways, each of which has limitations. A common approach is simply to disregard spatial variation in demography by applying estimates obtained from a single population to the entire area of interest (see review by Zeigler et al. [2013]). This strategy, however, has the potential to produce biased viability estimates if the population is not representative of other populations in the target area. It can also overestimate viability because including variation tends to decrease viability (Morris and Doak 2002). A second approach is to account implicitly for spatial variation in demography by combining data from multiple populations into a single estimate of each demographic rate (e.g., Doak et al. 1994, 
McGowan et al. 2017). Although resulting viability estimates may be reasonable across all populations, important site-specific differences in viability that can affect population dynamics locally and regionally are likely to be overlooked (e.g., source-sink dynamics; Pulliam 1988). Further, pooling data across populations ignores information about the relative location of populations, which is fundamental to characterizing spatial patterns and understanding the processes that govern these patterns. A third approach is to account explicitly for spatial variation in demography by characterizing rates of multiple populations and incorporating locations and other spatial information about these populations (Dunning et al. 1995, Turner et al. 1995, Reed et al. 2002, Beissinger et al. 2006). This approach, typically referred to as spatially explicit PVA, has the potential to provide the most reliable estimates of viability, although its data requirements are high, which can limit its application. For example, many early spatially explicit PVAs were a hybrid of the three approaches outlined above; they considered the effects of population spatial structure (e.g., location, size, and habitat features) on viability but relied on only one set of demographic rates for all locations (LaHaye et al. 1994, Lamberson et al. 1994, Akçakaya and Atwood 1997, McCarthy et al. 2000) or a few different sets that varied with environmental features (Pulliam et al. 1992) or patch sizes (Hokit and Branch 2003). More recently, some PVAs have incorporated spatial variation in demographic rates explicitly by estimating rates for a few discrete populations (e.g., Hernández-Matías et al. 2013, Bevacqua et al. 2015). Nevertheless, when a large number of populations are of interest or a species' distribution is relatively large and continuous, obtaining estimates from enough populations to reveal spatial patterns in demography at useful resolutions can be challenging. As such, these types of demographic data are uncommon, especially for long-lived species.

Using spatial models to estimate demographic rates presents a potential solution to many of the limitations of current approaches that account for spatial variation in PVAs. Spatial models include information about the location of each object of interest (e.g., individual, habitat patch, or population) as well as the relationships to objects in adjoining locations to inform parameter estimates for each location (Haining 1990, Cressie 1993). The ability to leverage data from surrounding locations not only can improve precision of estimates for locations where data are limited but it also allows parameters to be estimated for locations with no data. Consequently, spatial models can produce spatially explicit estimates across an entire area of interest, which can reveal spatial patterns that provide novel and important insights into population dynamics and other landscape-level processes. These capabilities can be especially important for modeling demographic rates derived from capture-recapture methods because studies employing these methods are labor intensive, so data tend to be sparse (McKelvey and Pearson 2001, Litt and Steidl 2010).

Despite the potential value of spatial models for demography, they had received little attention for this purpose until Saracco et al. (2010) developed a hierarchical, spatial autoregressive population model to produce spatially explicit estimates of demographic rates using capture-recapture data from locations distributed across a species' range.
Although they developed their model for survival and residency probabilities, their model provides a flexible framework that allows the underlying population model to be substituted with other population models that can yield spatially explicit estimates of the full array of demographic rates that are needed for PVA.

In this paper, we modify and extend the approach of Saracco et al. (2010) to develop a PVA that provides spatially explicit estimates of viability. Specifically, we describe an analytical approach to produce spatially explicit estimates of survival, transition, and recruitment rates at relatively fine spatial scales across a large spatial extent. We combine local estimates of these rates in stage-structured population models to estimate rates of population change throughout the focal area. We then use estimates of the rate of population change (and its uncertainty) to forecast local abundance and estimate viability. We apply the model to demographic data for the Sonoran desert tortoise (Gopherus morafkai), a species thought to be declining in response to an array of threats that includes habitat loss, invasive species, disease, and climate change (Zylstra et al. 2013, Gray and Steidl 2015, USFWS 2015). We examine the resulting spatial patterns in demographic rates, rates of population change, and viability for desert tortoises across their range in Arizona to illustrate the types of insights into the spatial structure and population dynamics that the model can provide. Finally, we discuss the implications of our findings for conservation, management, and research at multiple spatial scales.

\section{PVA MODEL}

The PVA model combines a spatial hierarchical model for estimating survival and transition rates, a spatial model for estimating juvenile recruitment (hereafter, recruitment), a stage-structured population model that integrates local demographic rates into estimates of rate of population change, and a simulation model that forecasts viability using the estimated rate of population change and its uncertainty. To implement the PVA model, we assume that the target species has two distinct life stages (i.e., juveniles and adults), that its populations are distributed continuously across a focal area, that demographic data are available from multiple locations throughout the focal area, that demographic rates are constant over time and identical for all individuals of the same life stage from the same location, and that individuals remain in the same general location throughout their life. The model can be modified easily to accommodate changes in one or more of these assumptions (e.g., increasing the number of stage or age classes, allowing demographic rates to vary with time, or modeling discrete populations). To apply the model, we employ Bayesian methods to estimate survival, transition, and recruitment and use the resulting posterior distributions to propagate uncertainty into estimates of rates of population change and viability.

\section{Survival and juvenile-to-adult transitions}

The model to estimate survival of juveniles and adults and the rate at which juveniles transition to adults relies on capture-recapture data. Data are assembled into an 
encounter history for each individual, which indicates for each year of the study if the individual was observed as a juvenile or adult, if it was not observed, or if a location was not surveyed. We estimate survival and transition rates from these encounter histories using a state-space, multi-state, population model (Calvert et al. 2009, Converse et al. 2012, Kéry and Schaub 2012). State-space models are hierarchical models that decompose encounter histories into two processes: the state process, which describes the ecological events that govern an individual's state (i.e., survival and transition), and the observation process, which describes factors that govern the ability to observe the state process (i.e., detection probability). We develop the state-space model for $i=1, \ldots, N$ encounter histories, $j=1, \ldots, M$ locations, and $t=1, \ldots, T$ years.

We describe the state process in terms of the true state of individual $i$ at location $j$ at time $t, z_{i, j, t}$. An individual can exist in one of three states: alive as a juvenile, alive as an adult, or dead. We model the state of an individual, $z_{i, j, t}$, as conditional on its state during the previous time period, $z_{i, j, t-1}$ and represent the state process as a categorical random variable:

$$
z_{i, j, t} \mid z_{i, j, t-1} \sim \operatorname{Cat}\left(\mathbf{s}_{j, z_{i, j, t-1}}\right)
$$

where $\mathbf{s}_{j, z_{i, j, t-1}}$ is a vector of state-transition probabilities for individual $i$ at location $j$ at time $t$, given that the individual was in state $z_{i, j, t-1}$ at time $t-1$. We describe state-transition probabilities $\left(\mathbf{s}_{j, z_{i, j, t-1}}\right)$ as a function of juvenile survival $\left(\phi_{\mathrm{juv}, j}\right)$, adult survival $\left(\phi_{\mathrm{ad}, j}\right)$, and the rate at which juveniles transitioned to adults $\left(\psi_{j}\right)$ :

$$
\left.\begin{array}{c}
\text { juvenile } \\
z_{i, j, t-1} \\
\begin{array}{c}
\text { juvenile } \\
\text { adult } \\
\text { dead }
\end{array}
\end{array} \quad \begin{array}{ccc}
z_{i, j, t} & \text { adult } & \text { dead } \\
\phi_{\text {juv }, j}\left(1-\psi_{j}\right) & \phi_{\text {juv }, j} \psi_{j} & 1-\phi_{\text {juv }, j} \\
0 & \phi_{\mathrm{ad}, j} & 1-\phi_{\mathrm{ad}, j} \\
0 & 0 & 1
\end{array}\right)
$$

For example, the state of an individual that was alive as a juvenile at location $j$ during the previous year is modeled as a multinomial random variable with a probability of surviving and remaining in the juvenile state equal to $\phi_{\mathrm{juv}, j}\left(1-\psi_{j}\right)$, a probability of surviving and transitioning into the adult state equal to $\phi_{\mathrm{juv}, j} \psi_{j}$, and a probability of not surviving equal to $1-\phi_{\mathrm{juv}, j}$. Because estimates are conditional on time of first capture $\left(t=f_{i}\right)$, the state of individual $i$ at time $f_{i}\left(z_{i, j, f_{i}}\right)$ is juvenile or adult with a probability of one. Additionally, when a juvenile transitions to an adult between time $t-1$ and $t$, we assume that it survives the time interval as a juvenile and then transitions to an adult as opposed to transitioning immediately to an adult and then surviving the interval as an adult.

We describe the observation process in terms of the observed state of individual $i$ at location $j$ at time $t, y_{i, j, t}$. An individual can be observed as a juvenile, observed as an adult, or not observed. We model the observed state $\left(y_{i, j, t}\right)$ as conditional on the latent state $\left(z_{i, j, t}\right)$ and assume that captured adults are not classified incorrectly as juvenile and vice versa. We represent the observation process as a categorical random variable

$$
y_{i, j, t} \mid z_{i, j, t} \sim \operatorname{Cat}\left(\mathbf{d}_{z_{i, j, t}}\right)
$$

where $\mathbf{d}_{z_{i, j, t}}$ is a vector of detection probabilities for individual $i$ at location $j$ at time $t$, given that the individual is in state $z_{i, j, t}$. We assume that detection probabilities depend only on the state of the individual; thus, we specify detection probabilities $\left(\mathbf{d}_{z_{i, j, t}}\right)$ as

$$
\begin{aligned}
& y_{i, j, t} \\
& \text { juvenile adult not observed } \\
& z_{i, j, t} \begin{array}{c}
\text { juvenile } \\
\text { adult } \\
\text { dead }
\end{array}\left(\begin{array}{ccc}
p_{\text {juv }} & 0 & 1-p_{\text {juv }} \\
0 & p_{\text {ad }} & 1-p_{\text {ad }} \\
0 & 0 & 1
\end{array}\right)
\end{aligned}
$$

where $p_{\text {juv }}$ and $p_{\text {ad }}$ represent detection probabilities for juveniles and adults, respectively.

We describe variation in survival $\left(\phi_{z, j}\right)$ and transition rates $\left(\psi_{j}\right)$ with logit-linear models. We assume that survival of an individual depends on its latent state and location

$$
\operatorname{logit}\left(\phi_{z, j}\right)=\mu_{z}+u_{z, j}
$$

and that probability of a juvenile transitioning to an adult depends on location

$$
\operatorname{logit}\left(\psi_{j}\right)=\alpha+v_{j}
$$

where $\mu_{z}$ is mean state-specific survival, $\alpha$ is mean transition rate, and $u_{z, j}$ and $v_{j}$ are random effects with means of zero and are assumed to be spatially autocorrelated.

We describe spatial dependence among elements of each vector of random effects (i.e., $\mathbf{u}_{z}=\left[u_{z, 1}, \ldots, u_{z, \mathrm{M}}\right]$ and $\mathbf{v}=\left[v_{1}\right.$, $\left.\ldots, v_{M}\right]$ ) with conditional autoregressive (CAR) models that assume the expectation of a value at a location is conditional on values at nearby locations (Ver Hoef et al. 2018). We use the intrinsic version of the CAR model (Besag and Kooperberg 1995, Thomas et al. 2014) for $u_{z, j}$ and $v_{j}$

$$
u_{z, j} \mid \mathbf{u}_{z,-j} \sim \operatorname{Norm}\left(\frac{1}{n_{j}} \sum_{k \in \mathbf{N}_{j}} u_{k}, \frac{\sigma_{\phi_{z}}^{2}}{n_{j}}\right)
$$

and

$$
v_{j} \mid \mathbf{v}_{-j} \sim \operatorname{Norm}\left(\frac{1}{n_{j}} \sum_{k \in \mathbf{N}_{j}} v_{k}, \frac{\sigma_{\psi}^{2}}{n_{j}}\right)
$$

where $u_{z, j}$ and $v_{j}$ are normally distributed with conditional mean given by the average of values of $n_{j}$ neighbors within the geographic neighborhood $\mathbf{N}_{j}$ of location $j$ (i.e., $n_{j}=\operatorname{dim}\left[\mathbf{N}_{j}\right]$ ) and conditional variance given by $\sigma_{\phi_{z}}^{2}$ or $\sigma_{\psi}^{2}$, which are variance parameters for $\phi_{z}$ and $\psi$ that serve as measures of spatial variation for each parameter, divided by $n_{j}$. CAR models are suited ideally for discrete spatial units, so we discretize the focal area into contiguous units by overlaying a regular grid.

\section{Recruitment}

The model to estimate recruitment relies on data appropriate for estimating the number of one-year-old females 
produced per adult female per year. Locations with recruitment estimates need not be the same locations where capture-recapture data are available. We describe variation in recruitment $\left(R_{j}\right)$ with log-linear models for $j=1, \ldots, M$ locations. We assume that recruitment follows a log-normal distribution and depends only on location

$$
\log \left(R_{j}\right)=\rho+w_{j}
$$

where $\rho$ is mean recruitment on the $\log$ scale and $w_{j}$ is a random effect with a mean of zero and is assumed to be spatially autocorrelated.

We describe spatial dependence among elements of the vector of random effects (i.e., $\mathbf{w}=\left[w_{1}, \ldots, w_{M}\right]$ ) with an intrinsic version of the CAR model

$$
w_{j} \mid \mathbf{w}_{-j} \sim \operatorname{Norm}\left(\frac{1}{n_{j}} \sum_{k \in \mathbf{N}_{j}} w_{k}, \frac{\sigma_{R}^{2}}{n_{j}}\right)
$$

where $w_{j}$ is normally distributed with conditional mean given by the average of values of $n_{j}$ neighbors within the geographic neighborhood $\mathbf{N}_{j}$ of location $j$ (i.e., $n_{j}=\operatorname{dim}\left[\mathbf{N}_{j}\right]$ ) and conditional variance given by $\sigma_{R}^{2}$, which is the variance parameter for $R$ that serves as a measure of spatial variation for the parameter, divided by $n_{j}$. We implement the CAR model for recruitment in the same manner as those for survival and transition rates.

\section{Rate of population change}

We combine posterior distributions of survival, transition rates, and recruitment into a posterior distribution for the annual rate of population change for each grid cell $\left(\lambda_{j}\right)$. Specifically, within cell $j$, we sample the posterior distributions of each demographic rate $l=1, \ldots, L$ times under the assumption that demographic parameters are uncorrelated. For each iteration, $l$, we combine demographic rates into a $2 \times 2$ population projection matrix from which we estimate $\lambda_{j, l}$ as the dominant eigenvalue

$$
\left[\begin{array}{cc}
\phi_{\mathrm{juv}, j, l}\left(1-\psi_{j, l}\right) & R_{l} \\
\phi_{\mathrm{juv}, j, l} \psi_{j, l} & \phi_{\mathrm{ad}, j, l}
\end{array}\right] \Rightarrow \lambda_{j, l}
$$

The resulting $L$ estimates of $\lambda_{j, l}$ form the posterior distribution for each grid cell.

\section{Population viability}

We compute population viability for each grid cell as the probability that the population of a cell becomes locally extinct or extirpated $\left(P_{\mathrm{ex}}\right)$ within an arbitrary period that encompasses multiple generations of the focal species. For each grid cell, we create replicate populations with the same initial abundance and with age structures proportional to the stable-age distribution and allow populations to change each year according to $\lambda$ s drawn randomly from the posterior distribution for that grid cell. If a population falls below an arbitrary quasi-extirpation threshold, we consider it extirpated for the remainder of the projection. We estimate
$P_{\text {ex }}$ for each grid cell as the proportion of populations that were extirpated.

\section{Example: Sonoran Desert Tortoises}

We applied the PVA model to data for the Sonoran desert tortoise, a long-lived (lifespan $>30 \mathrm{yr}$ ), slow-growing (15$20 \mathrm{yr}$ to reach sexual maturity) species with generation times of about $25 \mathrm{yr}$ (Germano et al. 2002). Sonoran desert tortoises inhabit arid environments throughout western and southern Arizona in the United States and western Sonora in Mexico (USFWS 2015, Edwards et al. 2016). Our study focused on the portion of their geographic range in Arizona, an area of approximately 10.4 million ha $(61 \%$ of their total range; USFWS 2015) that is delimited by the Colorado River to the north and west, the limits of the Sonoran Desert to the northeast, and the international boundary to the south (Fig. 1).

We considered demographic data from 18 locations that spanned much of this portion of their range (Fig. 1). Data for estimating survival and transition rates were available for 16 locations (Appendix S1: Table S1; Averill-Murray et al. 2002, Zylstra et al. 2013), and data for estimating recruitment were available for five locations, three of which were at or near the locations used to estimate survival and transition rates (Appendix S1: Table S2; Campbell et al. 2015). We note that the two most northwestern locations occur in a hybrid zone between Sonoran and Mojave (G. agassizii) desert tortoises (McLuckie et al. 1999, Edwards et al. 2015). Plots at each location ranged in size from 0.93 to $3.9 \mathrm{~km}^{2}$ and were at elevations between 450 and 1,200 m. Vegetation on plots was predominantly lower Colorado River and Arizona upland subdivisions of Sonoran desert scrub, but some plots also included elements of Mojave desert scrub, juniper

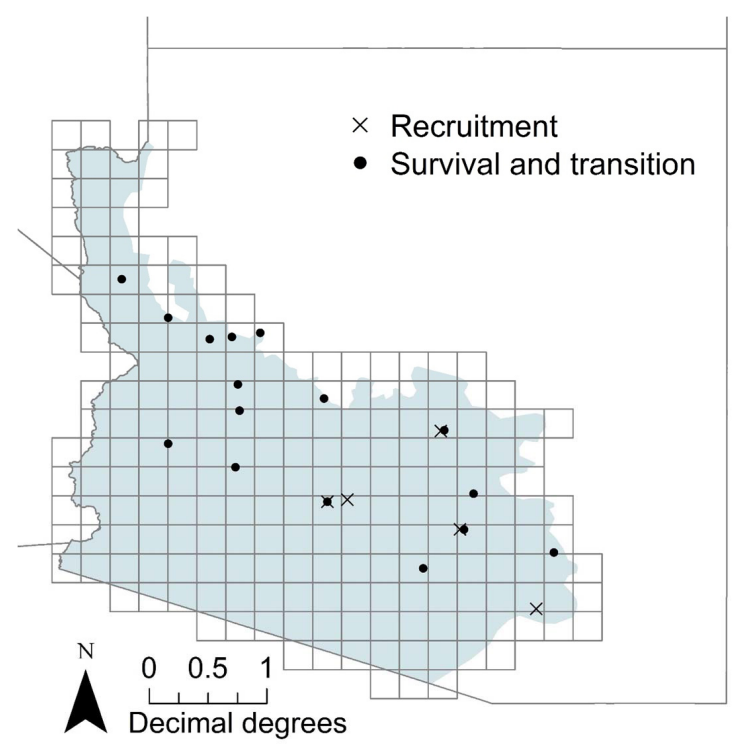

FIG. 1. Approximate range of Sonoran desert tortoises in Arizona, USA (shaded area) and sites where data were collected on survival and transition between life stages and recruitment. The range was divided into a grid of $2150.25^{\circ} \times 0.25^{\circ}$ cells to implement the spatial model. 
woodland, interior chaparral, or desert grassland (Brown 1994, Averill-Murray et al. 2002).

\section{Survival and juvenile-to-adult transitions}

There were 3-9 yr of capture-recapture data collected intermittently at each location between 1987 and 2008 (Appendix S1: Table S1). In years when locations were surveyed, observers searched plots up to five times between August and September when tortoises were most active. For each tortoise encountered, observers checked for previous marks, recorded sex, and measured midline carapace length (MCL), which was used to divide tortoises into juvenile $(<180 \mathrm{~mm})$ and adult $(\geq 180 \mathrm{~mm})$ size classes. If a tortoise was unmarked, observers filed a unique combination of notches in its marginal scutes (Cagle 1939). We used capture-recapture data to create a multi-state encounter history for each tortoise across $22 \mathrm{yr}$. Because of small sample sizes on most plots, we did not differentiate between encounter histories of males and females; therefore, we assumed that survival and transition rates for males and females were equal.

To implement the state-space, multi-state population model, we divided the species' range in Arizona into 215 contiguous units by overlaying a regular grid of cells sized $0.25^{\circ}$ latitude $\times 0.25^{\circ}$ longitude $(28 \times 24 \mathrm{~km}$; Fig. 1$)$. We chose this scale as a compromise between resolution of spatial patterns that could be supported reasonably by available demographic data and computational efficiency. Within this grid, we defined the geographic neighborhood of a cell $\left(\mathbf{N}_{j}\right)$ as adjacent cells in cardinal and ordinal directions (i.e., the eight surrounding cells).

We estimated survival and transition rates using Bayesian methods with OpenBUGS version 3.2.1 (Lunn et al. 2009). We established uniform prior distributions (Unif[0,1]) for $p_{z}$, $\phi_{z, j}$, and $\psi_{j}$, intrinsic Gaussian CAR prior distributions (Thomas et al. 2014) for spatial random effects $\left(u_{z, j}\right.$ and $\left.v_{j}\right)$, and gamma prior distributions (Gamma[0.1, 0.1]) for conditional precision parameters of the CAR models $\left(\tau_{\phi_{z}}\right.$ and $\tau_{\psi}$, where $\left.\tau=1 / \sigma^{2}\right)$. We obtained posterior distributions by sampling full-conditional distributions using Markov chain Monte Carlo methods (Gilks et al. 1996). Posterior distributions were based on 3,000 samples that were retained from three independent Markov chains of 30,000 samples after discarding the initial 27,000 samples and thinning the chain by three. These sampling parameters were adequate to obtain good model convergence based on inspecting chains visually and on Gelman-Rubin statistics $<1.1$ (Gelman et al. 2004).

\section{Recruitment}

Recruitment data were too limited geographically to produce spatially explicit estimates (Fig. 1); therefore, we did not use the recruitment model described above. Instead, we applied a single estimate across the entire range (i.e., $R$ rather than $R_{j}$ ). Without spatial variation in recruitment, spatial variation in viability reflected only spatial variation in survival and transition processes. We described methods for estimating range-wide recruitment of Sonoran desert tortoises elsewhere (Campbell et al. 2015); therefore, we summarize only the methodological information that is relevant for understanding how we integrated the recruitment estimate into our analysis. We calculated recruitment, $R$, as the product of four discrete components: proportion of females in the population that reproduced, number of female eggs produced per reproducing female per year, proportion of eggs that hatched successfully, and proportion of hatchlings that survived to the end of their first year. For the first two components, proportion of females that reproduced and number of female eggs produced, we used Bayesian generalized linear mixed models to obtain a posterior distribution of each component (proportion of females that reproduced, mean $=0.52,95 \%$ credible interval $=0.07-$ 0.94; number of female eggs produced, mean $=2.59,95 \%$ $\mathrm{CI}=1.53-3.80)$. Data for the latter two components, hatching success and hatchling survival, were sparse or non-existent (Appendix S1: Table S2). We represented the posterior distribution for each of these two components with a uniform distribution centered on ecologically plausible values of 0.8 and 0.3 for hatching success and hatchling survival, respectively, with bounds of the distributions established arbitrarily at the values \pm 0.2 . We then combined the posterior distributions of all four components to obtain a posterior distribution for recruitment with a mean of 0.32 females per female per year (95\% CI: $0.03-0.84)$.

\section{Rate of population change and population viability}

For each cell $j$, we sampled the posterior distributions of each demographic rate 3,000 times and combined them into 3,000 estimates of $\lambda_{j, l}$, which formed the posterior distribution for the rate of population change for each grid cell. We computed population viability $\left(P_{\mathrm{ex}}\right)$ for each grid cell over a period of $100 \mathrm{yr}$ using a quasi-extirpation threshold of 10 and 1,000 replicate populations. All populations in all grid cells started with the same abundance, which we varied between 25 and 10,000 (i.e., densities of $0.04-15.5 / \mathrm{km}^{2}$ ).

\section{RESULTS}

\section{Survival and transition rates}

We based estimates of survival and transition rates on capture-recapture data for 1,639 unique tortoises, $71 \%$ $(n=1,165)$ of which were first encountered as adults and $29 \%(n=474)$ as juveniles. Juveniles were more difficult to detect $\left(\hat{p}_{\text {juv }}=0.42,95 \%\right.$ credible interval: $\left.0.35-0.49\right)$ than adults $\left(\hat{p}_{\text {ad }}=0.75,0.72-0.78\right)$. For individuals first encountered as juveniles, $17 \%$ were encountered subsequently as adults.

Across their geographic range in Arizona, annual survival of adult tortoises $\left(\hat{\phi}_{\mathrm{ad}}\right)$ varied between 0.85 and 0.95 , annual survival of juveniles $\left(\hat{\phi}_{\text {juv }}\right)$ varied between 0.70 and 0.89 , and the annual rate at which juveniles transitioned to adults $(\hat{\psi})$ varied between 0.07 and 0.13 (Fig. 2). Precision of estimates was relatively high for survival of adults $(\mathrm{CV}=4.6 \%)$ and juveniles $(12.6 \%)$ but was low for transition rate $(52.7 \%$; Table 1$)$. Estimates of spatial variation in rates of juvenile and adult survival were similar $\left(\hat{\sigma}_{\phi_{\mathrm{juv}}}=1.08,0.50-2.06 ; \hat{\sigma}_{\phi_{\mathrm{ad}}}=1.07,0.69-1.53\right.$ ), both of which were higher than the estimate of spatial variation in transition rate $\left(\hat{\sigma}_{\psi}=0.83,0.21-2.32\right)$. 
Adult survival

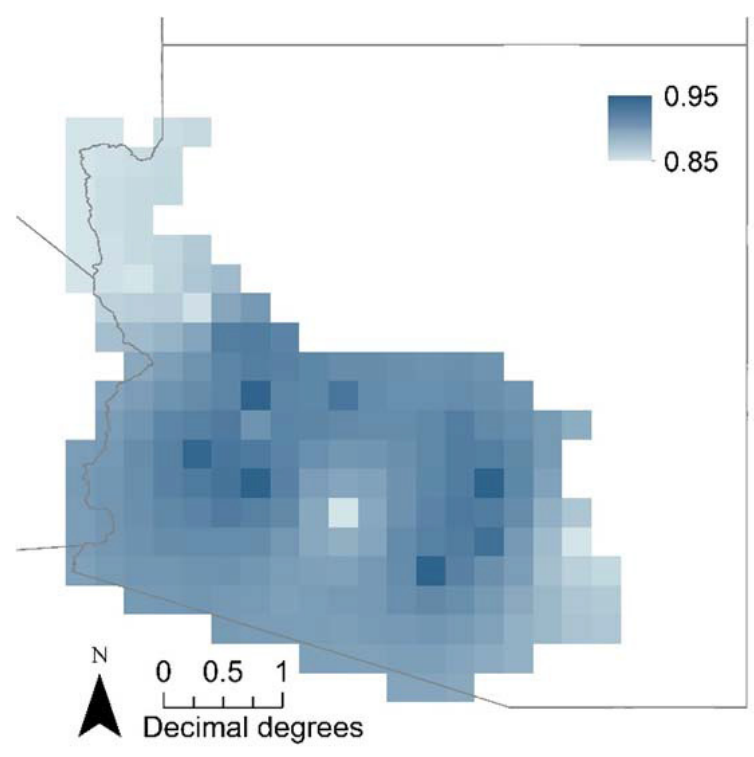

Juvenile survival

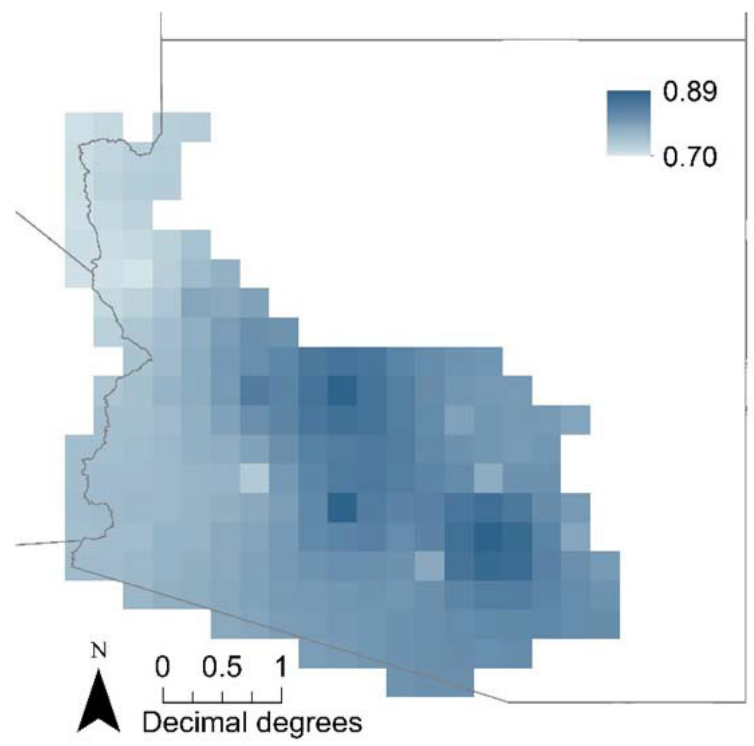

\section{Rate of population change}
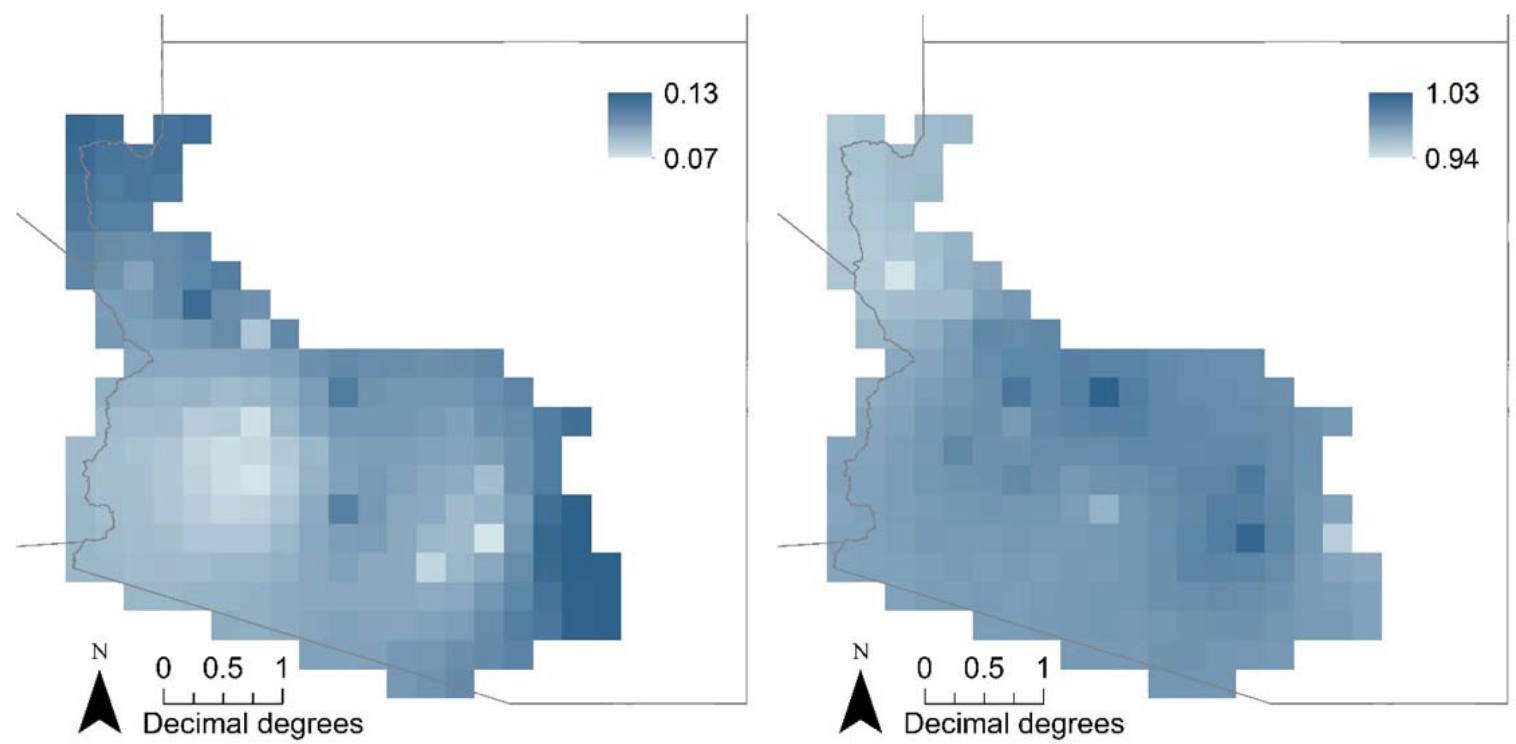

FIG. 2. Spatial patterns of mean adult and juvenile survival rates, juvenile-to-adult transition rates, and rates of population change of Sonoran desert tortoises across Arizona at a scale of $0.25^{\circ} \times 0.25^{\circ}$ grid cells. Estimates of survival and transition rates are based on encounter histories of 1,639 tortoises from 16 sites surveyed between 1987 and 2008. Rates of population change were based on a recruitment estimate of 0.32 females per female per year.

Survival of adults was lower in the far northwestern portion of Arizona and highest in the western- and eastern-central portions (Fig. 2). Survival of juveniles increased slightly from west to east, with areas of higher survival in the central portion of the state. Transition rates tended to vary negatively with adult survival and were higher in the northwestern and eastern portions of the state (Fig. 2). As expected, precision of all estimates was highest near sites where data were available and lowest in areas that are far from those sites (Appendix S2: Fig. S1).

\section{Rate of population change}

During the study period, rates of population change in Sonoran desert tortoises were generally stable across their range (mean $\hat{\lambda}=0.99$ ) when annual recruitment was 
TABLE 1. Summary of means, standard deviations, and coefficients of variation of posterior distributions of juvenile survival ( $\left.\phi_{\text {juv }}\right)$, adult survival $\left(\phi_{\mathrm{ad}}\right)$, juvenile-to-adult transition rate $(\psi)$, and rate of population change $(\lambda)$ from across the range of Sonoran desert tortoises in Arizona (see Fig. 2 and Appendix S2: Fig. S1).

\begin{tabular}{|c|c|c|c|c|c|c|}
\hline \multirow[b]{2}{*}{ Parameters } & \multicolumn{2}{|c|}{ Mean } & \multicolumn{2}{|c|}{ Standard deviation } & \multicolumn{2}{|c|}{ Coefficient of variation } \\
\hline & Mean & Range & Mean & Range & Mean & Range \\
\hline$\phi_{\mathrm{juv}}$ & 0.77 & $0.70-0.89$ & 0.10 & $0.02-0.16$ & 12.6 & $2.0-22.7$ \\
\hline$\phi_{\mathrm{ad}}$ & 0.91 & $0.85-0.95$ & 0.04 & $0.01-0.09$ & 4.6 & $0.7-10.2$ \\
\hline$\psi$ & 0.09 & $0.07-0.13$ & 0.05 & $0.01-0.10$ & 52.7 & $16.6-87.1$ \\
\hline$\lambda$ & 0.99 & $0.94-1.03$ & 0.06 & $0.03-0.09$ & 6.0 & $3.3-9.2$ \\
\hline
\end{tabular}

assumed to be 0.32 females per female. The rate of population change varied geographically between 0.94 and 1.03 , with about $4 \%$ of the range increasing $(\hat{\lambda} \geq 1.01)$ and $44 \%$ decreasing $(\hat{\lambda} \leq 0.99)$. The rate of population change tended to be higher in the central and eastern portions of Arizona and lower in the extreme northwestern part of the state (Fig. 2).

\section{Population viability}

As expected, $P_{\text {ex }}$ reflected initial abundance of local populations (Appendix S2: Fig. S2). Across all locations, average $P_{\text {ex }}$ increased from $0 \%$ when initial abundance was 5,000 to $56 \%$ when initial abundance was 25 . Spatial variation in $P_{\text {ex }}$ also increased as initial abundance decreased (Appendix S2: Fig. S2). When initial abundances were $>500, P_{\text {ex }}$ was near zero $(<0.05)$ across more than $85 \%$ of the entire range. When initial abundances were $\leq 500, P_{\mathrm{ex}}$ varied between zero and one across Arizona.

Spatial patterns in $P_{\text {ex }}$ suggested that populations in the northwest portion of the species' range were most vulnerable (Fig. 3). As initial abundances decreased, $P_{\mathrm{ex}}$ in this area increased, whereas $P_{\text {ex }}$ remained low across the remainder of the range. Nevertheless, when initial abundances decreased below 50, $P_{\text {ex }}$ also increased in the western and southern portions of the state (Fig. 3).

\section{DisCUSSION}

Spatial variation in demographic rates is common in animal populations (e.g., Ozgul et al. 2006, Ciannelli et al.
2007, Hernández-Matías et al. 2013, Zylstra et al. 2013) and can have important consequences for population dynamics and viability (Kareiva 1990, Watkinson and Sutherland 1995, Morris and Doak 2002). PVAs that consider spatial variation in underlying demographic rates explicitly, however, are rare (Zeigler et al. 2013), largely because the amount of data required to characterize demography over large spatial scales is considerable and the analytical methods to accommodate explicit spatial relationships in these data have been advanced only recently (Saracco et al. 2010). In this paper, we sought to address this deficiency by describing a spatially explicit PVA that leverages recent developments in hierarchical modeling, spatial models, and Bayesian analyses to estimate demography and viability at relatively fine spatial scales using data from locations distributed sparsely throughout a large focal area. Notably, our method differs from other spatially explicit PVAs in the way we treat spatial variation in the underlying demographic rates. Many spatial PVAs treat this type of fine-scale variation as a source of uncertainty that needs to be incorporated within a single estimate of viability at larger spatial scales to improve its reliability. In contrast, we treat spatial variation as an ecological feature of inherent interest, principally because this information can provide novel insights about spatial structure and dynamics of populations for conservation, management, and research at multiple spatial scales (e.g., grid cell, regional, species range).

At the smallest scale of our model (i.e., grid cell), estimates of demography and viability can provide insight into local population dynamics. For example, elasticity and sensitivity analyses on the scale of an individual grid cell can
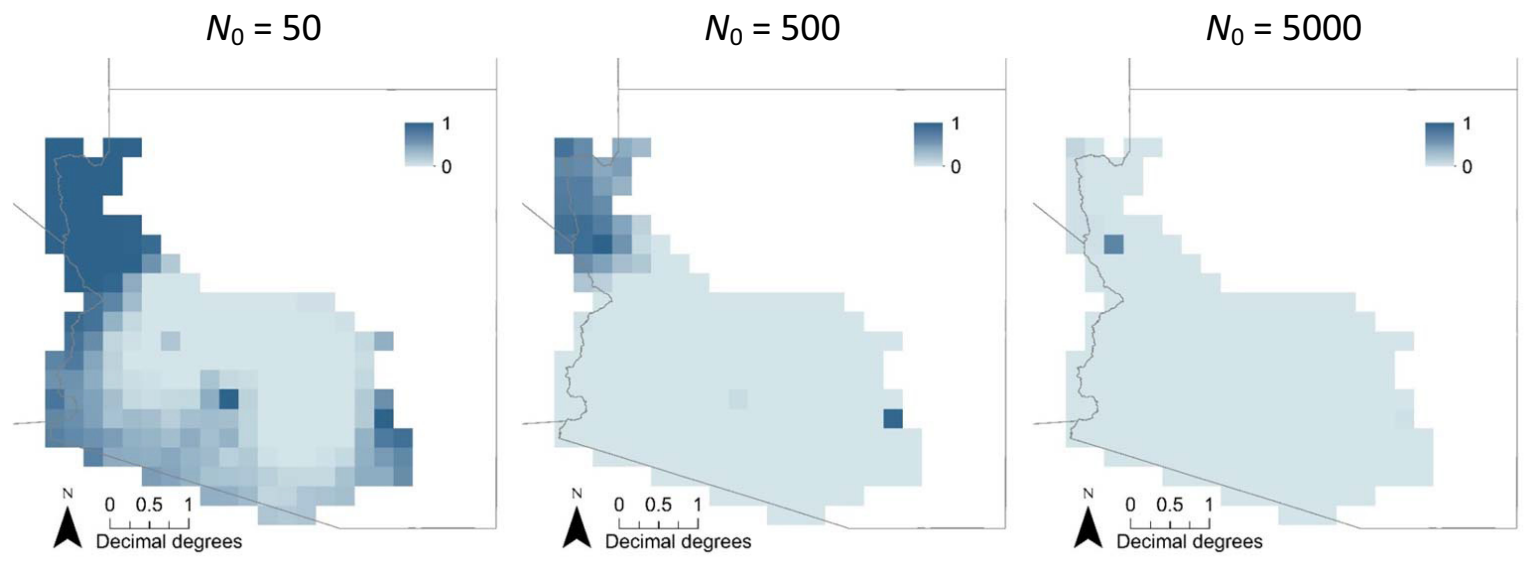

FIG. 3. Spatial patterns of probabilities of extirpation for Sonoran desert tortoises across their range in Arizona based on a recruitment estimate of 0.32 females per female per year and different initial population sizes $\left(N_{0}=50,500\right.$, and 5,000). 
identify specific demographic rates that can serve as targets for conservation efforts based on the magnitude of their effects on the rate of population change (Benton and Grant 1999, Caswell 2001). Similarly, perturbation analyses can inform how changes in threats or alternative management actions are likely to affect viability. Estimates of viability from individual grid cells also can be used to contrast areas with high and low viability to identify local factors that might explain these differences. Additionally, estimates at small scales provide a foundation for understanding population dynamics expressed at larger spatial scales. For example, a population that is predicted to have low viability based on its demographic rates can be stable or increasing if immigration from neighboring areas is high (Pulliam 1988). Consequently, managers would seek to protect adjacent areas because loss of nearby source populations could contribute to extirpation on a larger scale.

Spatially explicit estimates of demographic rates and viability also can reveal broad-scale patterns of spatial variation, which can illustrate the influence of regional-scale processes on population dynamics, provide a basis for developing research hypotheses about processes that give rise to these patterns, and identify scales most appropriate for management (Dunning et al. 1995, Rodenhouse et al. 1997). Across much of the range of the Sonoran desert tortoise in Arizona, for example, there was evidence of regional variation in survival and transition rates as well as small-scale variation among adjacent grid cells (Fig. 2). These patterns suggest that survival and transition rates are influenced, at least in part, by biological and physical processes operating at local to regional scales (Zylstra et al. 2013). There was also no clear association between juvenile and adult survival across much of their range, suggesting that demographic effects of biological and physical processes vary across life stages. Collectively, these patterns suggest that range-wide management efforts aimed at increasing demographic rates may not be an effective strategy for improving viability. Instead, local or regional efforts focused on increasing particular demographic rates might be a more reasonable strategy (e.g., increasing adult and juvenile survival in the northwestern portion of their range). Despite different spatial patterns among demographic rates, viability was relatively high across most of the geographic range except the northwestern portion. In this part of the range, lower survival of adult and juvenile tortoises resulted in lower viability (Figs. 2 and 3). We note, however, that in the northwestern part of the range, Sonoran desert tortoises likely hybridize with Mojave desert tortoises. Consequently, low viability of tortoises may be an artifact of using reproductive rates for Sonoran desert tortoises in an area where rates of Mojave desert tortoises, which are higher, may be more appropriate (Averill-Murray et al. 2014, Campbell et al. 2015).

Our approach to PVA also can be used to estimate viability at regional or range-wide scales. Specifically, information about movements of individuals among grid cells (i.e., dispersal rules) can be included to aggregate demographic information from grid cells into a single estimate of viability (Dunning et al. 1995, Reed et al. 2002, Beissinger et al. 2006). Although this type of large-scale PVA is used often for determining the conservation status of a species (e.g., Doak et al. 1994, McGowan et al. 2017), aggregating spatially explicit estimates for this goal is beneficial only to the extent that incorporating spatial variation improves the reliability of species-level estimates of viability. The greater utility of spatially explicit estimates for species conservation will likely come from understanding how viability and dynamics of populations vary geographically in relation to each other and to landscape features and threats.

We focused on modeling local population viability by accounting for spatial variation in demographic rates using information from adjacent areas when arranged as a grid. We acknowledge, however, that spatial variation in population processes could be incorporated in other ways; therefore, we provide five examples of possible modifications. First, environmental variables alone or in conjunction with spatial effects (i.e., autocorrelation) can be included in the models of demographic rates (sensu Guisan and Thuiller 2005). Although including environmental variables can provide insight into mechanisms that explain spatial patterns and allow forecasting as conditions change, the resulting models may describe patterns no better or even worse than purely spatial models (Bahn and McGill 2007). Second, the model could be extended to allow for correlations among demographic parameters within years (e.g., Doak et al. 1994, Schaub et al. 2013). For the desert tortoise, we assumed demographic parameters were independent. Violation of this assumption, however, could underestimate variance in the rate of population change, which would overestimate population viability (Morris and Doak 2002). Third, our estimates of viability for desert tortoises were based on the same initial abundances for all grid cells. Although this approach provides a relative rank of viability among grid cells, it does not predict reliably what is likely to occur within cells (Turner et al. 1995, Beissinger and Westphal 1998, Ralls et al. 2002, Reed et al. 2002). Using empirically based estimates of local abundance that vary on the same spatial scale as demographic rates $\left(0.25^{\circ} \times 0.25^{\circ}\right.$ in our example) would better reflect the actual extirpation risk at a particular grid cell and would more accurately represent spatial variation in viability of the target species. Fourth, we defined the neighborhood for autocorrelation in the model for desert tortoises as adjacent cells in the cardinal and ordinal directions. Although this is a common approach, neighborhoods could be established to capture different spatial relationships. For example, if evidence suggests that autocorrelation occurs across greater distances, the neighborhood could be defined to include neighbors up to two or more grid cells away and weights in the CAR model can be assigned to cells according to their distance from the focal cell. Finally, grid-cell sizes can be altered to accommodate different scales or to examine the sensitivity of population viability estimates to spatial resolution. In our case study, estimates of demographic parameters and rate of population change and their spatial patterns remained largely consistent across three different cell sizes (Appendix S3). There was, however, a slight increase in variation of demographic rates as cell size increased, which resulted in nominally higher extirpation probabilities (Appendix S3).

As with all PVAs, the reliability and ultimate value of the results from our model depend on the quality and amount 
of data (Beissinger and Westphal 1998). Even though spatial models use data more efficiently, data requirements for population-based studies of long-lived species at this scale are still high because detailed demographic data are required for multiple years at many locations. Consequently, such data are only available infrequently, especially for endangered species (Tear et al. 1995, Beissinger and Westphal 1998, Morris et al. 2002, Zeigler et al. 2013). For Sonoran desert tortoises, recruitment data were too limited to produce spatially explicit estimates; therefore, we explored the effects of varying range-wide recruitment estimates on the magnitude and spatial patterns of viability (Campbell et al. 2015; Appendix S4). The sensitivity of viability to differences in recruitment underscores the value of site-specific information. In contrast, data for estimating survival and transition rates were considerably more robust. Nevertheless, even these data were sparse in some regions, which can lead to estimates for large geographic regions that are informed by data from few locations. Sparse data also result in propagation of autocorrelation over large distances, which increases uncertainty in demographic rates far from locations with data (Appendix S2: Fig. S1) and increases predicted risk of extirpation in these areas (Morris and Doak 2002). Despite these limitations, our results illustrate that viability can still be explored and insights gained with sparse data, which will be the rule for rare species with broad distributions. Fortunately, data collected over large temporal and spatial scales are increasing in availability for many species through continent-scale monitoring projects (DeSante and Kaschube 2009), largescale citizen-science projects (Dickinson et al. 2012, McKinley et al. 2015), and data sharing among researchers (Crall et al. 2006, Duke 2006). As these types of data become more common, we anticipate a growth in efforts to relate spatial patterns in demography and viability to largescale phenomena with effects that are potentially complex and spatially varying.

\section{ACKNOWLEDGMents}

This work was funded by the U.S. Fish and Wildlife Service and the Arizona Game and Fish Department. We thank C. A. Jones from the Arizona Game and Fish Department for supplying much of the data we used in the analysis and the dozens of surveyors who collected these data in the field. The findings and conclusions provided in this article are those of the authors and do not necessarily represent the view of the U.S. Fish and Wildlife Service.

\section{Literature Cited}

Akçakaya, H. R., and J. L. Atwood. 1997. A habitat-based metapopulation model of the California gnatcatcher. Conservation Biology 11:422-434.

Akçakaya, H. R., and P. Sjögren-Gulve. 2000. Population viability analyses in conservation planning: an overview. Ecological Bulletins 48:9-21.

Averill-Murray, R. C., A. P. Woodman, and J. M. Howland. 2002. Population ecology of the Sonoran desert tortoise in Arizona. Pages 109-134 in T. R. Van Devender, editor. The Sonoran desert tortoise: natural history, biology, and conservation. University of Arizona Press, Tucson, Arizona, USA.

Averill-Murray, R. C., L. J. Allison, and L. L. Smith. 2014. Nesting and reproductive output among North American tortoises.
Pages 110-117 in D. C. Rostal, E. D. McCoy, and H. Mushinsky, editors. Biology and conservation of North American tortoises. Johns Hopkins University Press, Baltimore, Maryland, USA.

Bahn, V., and B. J. McGill. 2007. Can niche-based distribution models outperform spatial interpolation? Global Ecology and Biogeography 16:733-742.

Beissinger, S. R., and D. R. McCullough, editors. 2002. Population viability analysis. University of Chicago Press, Chicago, Illinois, USA.

Beissinger, S. R., and M. I. Westphal. 1998. On the use of demographic models of population viability in endangered species management. Journal of Wildlife Management 62:821-841.

Beissinger, S. R., J. R. Walters, D. G. Catanzaro, K. G. Smith, J. B. Dunning, Jr., S. M. Haig, B. R. Noon, and B. M. Stith. 2006. Modeling approaches in avian conservation biology and the role of field biologists. Ornithological Monographs 59:1-56.

Benton, T. G., and A. Grant. 1999. Elasticity analysis as an important tool in evolutionary and population ecology. Trends in Ecology and Evolution 14:467-471.

Besag, J., and C. Kooperberg. 1995. On conditional and intrinsic autoregressions. Biometrika 82:733-746.

Bevacqua, D., P. Meliá, M. Gatto, and G. A. De Leo. 2015. A global viability assessment of the European eel. Global Change Biology 21:3323-3335.

Brown, D. E., editor. 1994. Biotic communities: southwestern United States and northwestern Mexico. University of Utah Press, Salt Lake City, Utah, USA.

Cagle, F. R. 1939. A system of marking turtles for future identification. Copeia 1939:170-173.

Calvert, A. M., S. J. Bonner, I. D. Jonsen, J. M. Flemming, S. J. Walde, and P. D. Taylor. 2009. A hierarchical Bayesian approach to multi-state mark-recapture: simulations and applications. Journal of Applied Ecology 46:610-620.

Campbell, S. P., R. J. Steidl, and E. R. Zylstra. 2015. Recruitment of desert tortoises (Gopherus agassizii and G. morafkai): a synthesis of reproduction and first-year survival. Herpetological Conservation and Biology 10:583-591.

Carroll, R., C. Augspurger, A. Dobson, J. Franklin, G. Orians, W. Reid, R. Tracy, D. Wilcove, and J. Wilson. 1996. Strengthening the use of science in achieving the goals of the Endangered Species Act: an assessment by the Ecological Society of America. Ecological Applications 6:1-11.

Caswell, H. 2001. Matrix population models. Second edition. Sinauer Associates, Sunderland, Massachusetts, USA.

Ciannelli, L., G. E. Dingsør, B. Bogstad, G. Ottersen, K. Chan, H. Gjøsæter, J. E. Stiansen, and N. C. Stenseth. 2007. Spatial anatomy of species survival: effects of predation and climate-driven environmental variability. Ecology 88:635-646.

Converse, S. J., J. A. Royle, and R. P. Urbanek. 2012. Bayesian analysis of multi-state data with individual covariates for estimating genetic effects on demography. Journal of Ornithology 152:S561S572.

Crall, A. W., L. A. Meyerson, T. J. Stohlgren, C. S. Jarnevich, G. J. Newman, and J. Graham. 2006. Show me the numbers: what data currently exist for non-native species in the USA? Frontiers in Ecology and the Environment 4:414-418.

Cressie, N. A. 1993. Statistics for spatial data, Revised edition. John Wiley and Sons, New York, New York, USA.

DeSante, D. F., and D. R. Kaschube. 2009. The monitoring avian productivity and survivorship (MAPS) program 2004, 2005, and 2006 report. Bird Populations 9:86-169.

Dickinson, J. L., J. Shirk, D. Bonter, R. Bonney, R. L. Crain, J. Martin, T. Phillips, and K. Purcell. 2012. The current state of citizen science as a tool for ecological research and public engagement. Frontiers in Ecology and the Environment 10:291-297.

Doak, D., P. Kareiva, and B. Klepetka. 1994. Modeling population viability for the desert tortoise in the western Mojave Desert. Ecological Applications 43:446-460. 
Duke, C. S. 2006. Data: share and share alike. Frontiers in Ecology and the Environment 4:395.

Dunning, J. B., D. J. Stewart, B. J. Danielson, B. R. Noon, T. L. Root, R. H. Lamberson, and E. E. Stevens. 1995. Spatially explicit population models: current forms and future uses. Ecological Applications 5:3-11.

Edwards, T., K. H. Berry, R. D. Inman, T. C. Esque, K. E. Nussear, C. A. Jones, and M. Culver. 2015. Testing taxon tenacity of tortoises: evidence for a geographical selection gradient at a secondary contact zone. Ecology and Evolution 5:2095-2114.

Edwards, T., A. E. Karl, M. Vaughn, P. C. Rosen, C. M. Torres, and R. W. Murphy. 2016. The desert tortoise trichotomy: Mexico hosts a third, new sister-species of tortoise in the Gopherus morafkai-G. agassizii group. ZooKeys 562:131-158.

Gelman, A., J. B. Carlin, H. S. Stern, and D. B. Rubin. 2004. Bayesian data analysis. Second edition. Chapman \& Hall/CRC, Boca Raton, Florida, USA.

Germano, D. J., F. H. Pough, D. J. Morafka, E. M. Smith, and M. J. Demlong. 2002. Growth of desert tortoises: implications for conservation and management. Pages 265-288 in T. R. Van Devender, editor. The Sonoran desert tortoise: natural history, biology, and conservation. University of Arizona Press, Tucson, Arizona, USA.

Gilks, W. R., S. Richardson, and D. Spiegelhalter. 1996. Markov chain Monte Carlo in practice. Chapman \& Hall/CRC Press, Boca Raton, Florida, USA.

Gray, K. M., and R. J. Steidl. 2015. A plant invasion affects the condition but not density or population structure of a vulnerable reptile. Biological Invasions 17:1979-1988.

Guisan, A., and W. Thuiller. 2005. Predicting species distribution: offering more than simple habitat models. Ecology Letters 8:9931009.

Haining, R. 1990. Spatial data analysis in the social and environmental sciences. Cambridge University Press, Cambridge, UK.

Hernández-Matías, A., et al. 2013. From local monitoring to broad-scale viability assessment: a case study for the Bone1li's Eagle in Western Europe. Ecological Monographs 83: 239-261.

Hokit, D. G., and L. C. Branch. 2003. Associations between patch area and vital rates: consequences for local and regional populations. Ecological Applications 13:1060-1068.

Kareiva, P. 1990. Population dynamics in spatially complex environments: theory and data. Philosophical Transactions of the Royal Society B 330:175-190.

Kéry, M., and M. Schaub. 2012. Bayesian population analysis using WinBUGS: a hierarchical perspective. Academic Press, Waltham, Massachusetts, USA.

LaHaye, W. S., R. J. Gutiérrez, and H. R. Akçakaya. 1994. Spotted owl metapopulation dynamics in southern California. Journal of Animal Ecology 63:775-785.

Lamberson, R. H., B. R. Noon, C. Voss, and K. S. McKelvey. 1994. Reserve design for territorial species: the effects of patch size and spacing on the viability of the Northern Spotted Owl. Conservation Biology 8:185-195.

Litt, A. R., and R. J. Steidl. 2010. Improving estimates of abundance by aggregating sparse capture-recapture data. Journal of Agricultural, Biological, and Environmental Statistics 15:228-247.

Lunn, D., D. Spiegelhalter, A. Thomas, and N. Best. 2009. The BUGS project: evolution, critique and future directions. Statistics in Medicine 28:3049-3067.

McCarthy, M. A., D. B. Lindenmayer, and H. P. Possingham. 2000. Testing spatial PVA models of Australian treecreepers (Aves: Climacteridae) in fragmented forest. Ecological Applications 10:1722-1731.

McGowan, C. P., N. Allan, J. Servoss, S. Hedwall, and B. Wooldridge. 2017. Incorporating population viability models into species status assessment and listing decisions under the U.S. Endangered Species Act. Global Ecology and Conservation 12:119-130.
McKelvey, K. S., and D. E. Pearson. 2001. Population estimation with sparse data: the role of estimators versus indices revisited. Canadian Journal of Zoology 79:1754-1765.

McKinley, D. C., et al. 2015. Investing in citizen science can improve natural resource management and environmental protection. Issues in Ecology 19:1-27.

McLuckie, A. M., T. Lamb, C. R. Schwalbe, and R. D. McCord. 1999. Genetic and morphometric assessment of an unusual tortoise (Gopherus agassizii) population in the Black Mountains of Arizona. Journal of Herpetology 33:36-44.

Menges, E. S. 2000. Population viability analyses in plants: challenges and opportunities. Trends in Ecology \& Evolution 15:51-56.

Morris, W. F., and D. F. Doak. 2002. Quantitative conservation biology: theory and practice of population viability analysis. Sinauer Associates, Sunderland, Massachusetts, USA.

Morris, W. F., P. L. Bloch, B. R. Hudgens, L. C. Moyle, and J. R. Stinchcombe. 2002. Population viability analysis in endangered species recovery plans: past use and future improvements. Ecological Applications 12:708-712.

Ozgul, A., K. B. Armitage, D. T. Blumstein, and M. K. Oli. 2006. Spatiotemporal variation in survival rates: implications for population dynamics of yellow-bellied marmots. Ecology 87:1027-1037.

Pulliam, H. R. 1988. Sources, sinks, and population regulation. American Naturalist 132:652-661.

Pulliam, H. R., J. B. Dunning Jr., and J. Liu. 1992. Population dynamics in complex landscapes: a case study. Ecological Applications 2:165-177.

Ralls, K., S. R. Beissinger, and J. F. Cochrane. 2002. Guidelines for using population viability analysis in endangered-species management. Pages 521-550 in S. R. Beissinger and D. R. McCullough, editors. Population viability analysis. University of Chicago Press, Chicago, Illinois, USA.

Reed, J. M., L. S. Mills, J. B. Dunning Jr., E. S. Menges, K. S. McKelvey, R. Frye, S. Beissinger, M.-C. Anstett, and P. Miller. 2002. Emerging issues in population viability analysis. Conservation Biology 16:7-19.

Rodenhouse, N. L., T. W. Sherry, and R. T. Holmes. 1997. Sitedependent regulation of population size: a new synthesis. Ecology 78:2025-2042.

Saracco, J. F., J. A. Royle, D. F. DeSanter, and B. Gardner. 2010. Modeling spatial variation in avian survival and residency probabilities. Ecology 91:1885-1891.

Schaub, M., H. Jakober, and W. Stauber. 2013. Strong contribution of immigration to local population regulation: evidence from a migratory passerine. Ecology 94:1828-1838.

Schemske, D. W., B. C. Husband, M. H. Ruckelshaus, C. Goodwillie, I. M. Parker, and J. G. Bishop. 1994. Evaluating approaches to the conservation of rare and endangered plants. Ecology 75:584-606.

Tear, T. H., J. M. Scott, P. H. Hayward, and B. Griffith. 1995. Recovery plans and the Endangered Species Act: are criticisms supported by data? Conservation Biology 9:182-195.

Thomas, A., N. Best, D. Lunn, R. Arnold, and D. Spiegelhalter. 2014. GeoBUGS user manual, version 3.2.3. http://www.openb ugs.net/Manuals/GeoBUGS/Manual.html

Turner, M. G., G. J. Arthaud, R. T. Engstrom, S. J. Hejl, J. Liu, S. Loeb, and K. McKelvey. 1995. Usefulness of spatially explicit population models in land management. Ecological Applications 5:12-16.

U.S. Fish and Wildlife Service (USFWS). 2015. Species status assessment for the Sonoran desert tortoise. Version 1.0, September 2015. U.S. Fish and Wildlife Service, Southwest Region, Albuquerque, New Mexico, USA.

Ver Hoef, J. M., E. E. Peterson, M. B. Hooten, E. M. Hanks, and M.-J. Fortin. 2018. Spatial autoregressive models for statistical inference from ecological data. Ecological Monographs 88: $36-59$.

Watkinson, A. R., and W. J. Sutherland. 1995. Sources, sinks, and pseudo-sinks. Journal of Animal Ecology 64:126-130. 
Zeigler, S. L., J. P. Che-Castaldo, and M. C. Neel. 2013. Actual and potential use of population viability analyses in recovery of plant species listed under the U.S. Endangered Species Act. Conservation Biology 27:1265-1278.
Zylstra, E. R., R. J. Steidl, C. A. Jones, and R. C. Averill-Murray. 2013. Spatial and temporal variation in survival of a rare reptile: a 22-year study of Sonoran desert tortoises. Oecologia 173: $107-116$.

\section{SUPPORTING INFORMATION}

Additional supporting information may be found online at: http://onlinelibrary.wiley.com/doi/10.1002/eap.1794/full

\section{Data Availability}

Data are available from the Dryad Digital Repository: https://doi.org/10.5061/dryad.v0q5035. 